\title{
Editorials
}

\section{Ropivacaine cardiac toxicity - not as troublesome as bupivacaine}

Brendan T. Finucane $\mathrm{MB}$ BCH BAO FRCPC FRCA

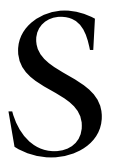

ERIOUS toxic reactions have been a problem with local anesthetic drugs since they were first discovered by Koller in $1884 .^{1}$ Several hundred local anesthetics have been tested during the last 100 years and very few have reached the marketplace. Lidocaine has been in clinical use for close to 60 years and is still the most widely used local anesthetic worldwide and remains one of the safest and most efficacious local anesthetic agents ever manufactured. One of lidocaine's main limitations is its short duration of action. Bupivacaine was introduced into clinical practice in 1967 and represented a new family of local anesthetics - the pipecholoxylidine derivatives. Initial reports about the safety and efficacy of bupivacaine were very promising. It is a very long acting local anesthetic, and there is a very clear separation between motor and sensory anesthesia in contrast to all previous local anesthetics. Bupivacaine was in clinical use for close to ten years before serious cardiac toxicity was reported. Several deaths were reported in obstetric patients in the United States in the 1970s. ${ }^{2}$ The search for a replacement for bupivacaine began in the 1980s. Ropivacaine was first tested in 1988 and appeared to have many of the blocking characteristics of bupivacaine but was much less toxic. ${ }^{3}$ Most local anesthetics are produced as racemic mixtures. Ropivacaine was the first synthetically produced local anesthetic that was a single enantiomer, and for that reason was predictably less toxic. It was first approved for clinical use in North America in 1996. The first reports of serious cardiac toxicity with ropivacaine were published in 2003. Two patients suffered cardiac arrest and one patient developed ventricular fibrillation following excessive doses of ropivacaine..$^{4-6}$
In this issue of the Canadian Journal of Anesthesia there is an additional report of ventricular fibrillation following an accidental intravascular injection of ropivacaine. ${ }^{7}$ There were some interesting aspects about this case that are worthy of comment. This case clearly illustrates the importance of monitoring patients when injecting potent local anesthetics. Regional anesthesia is frequently performed in 'block rooms' or 'holding areas' adjoining operating rooms where access to full monitoring is not always available. All patients undergoing regional anesthesia should have electrocardiogram (ECG) and blood pressure monitoring and pulse oximetry, as was performed in this case. This case also illustrates the importance of sedation and of having the patient's undivided attention when performing regional anesthesia.

It is interesting to note that all four patients who developed serious cardiac toxicity following ropivacaine were undergoing peripheral nerve blocks of the lower extremity, using nerve stimulation techniques. Three of these patients were elderly and received $0.75 \%$ solutions of ropivacaine and the toxic reactions reported were most likely due to accidental intravascular injections. Peripheral nerve blocks are being used more frequently worldwide for a number of reasons and electrical stimulation is frequently used to pinpoint the anatomic location of various nerves. Most injections are administered through needles for peripheral nerve blocks using a single injection technique. Once the anatomic location of a nerve has been identified using electrical stimulation, the position of the needle is fixed and the local anesthetic is injected, taking care not to move the needle. There is also a tendency to inject local anesthetics more rapidly under these circumstances because of the concern about

From the Department of Anesthesiology and Pain Medicine, University of Alberta, Edmonton, Alberta, Canada.

Address correspondence to: Dr. Brendan T. Finucane, University of Alberta, Department of Anesthesiology and Pain Medicine, Clinical Sciences Building 8-120, Edmonton, Alberta T6G 2G3, Canada. Phone: 780-407-2876; Fax: 780-407-7461;

E-mail: bfinucan@ualberta.ca 
patient movement as was illustrated in Gielen's case. ${ }^{7}$ It is particularly important to use a cardiovascular marker (epinephrine) under these circumstances. Ropivacaine is frequently injected without the addition of epinephrine because it is not enhanced by epinephrine and the plasma uptake of ropivacaine is minimally affected. ${ }^{8}$ Epinephrine was not added to the local anesthetic solution in three out of the four cases that developed serious cardiac toxicity following ropivacaine and was used in a reduced concentration in the fourth case $(1: 400,000)$. Epinephrine is an essential additive to all potent local anesthetics for major regional anesthesia, except when contraindicated.

Nerve stimulation techniques have not been universally adopted by regional anesthesia experts worldwide. Experienced artisans of the 'old school' of regional anesthesia do not use nerve stimulation for peripheral nerve blocks but instead prefer to rely on the paresthesia method to identify the location of the nerve. They then flood the anatomic region with the local anesthetic, moving the needle back and forth in a fanning motion. This approach may reduce the likelihood of injecting large doses of local anesthetics intravascularly. Whatever technique one uses, it is important to administer small incremental doses of local anesthetics (no more than $5 \mathrm{~mL}$ at a time) then pause and make verbal contact with the patient before proceeding with another increment. It is interesting to note that single injection, epidural techniques were frequently used by anesthesiologists in obstetric patients presenting for Cesarean section 25 years ago when Albright alerted us about bupivacaine cardiac toxicity. ${ }^{7}$

There seems to be some confusion among anesthesiologists about what constitutes a safe dose of ropivacaine. Most anesthesiologists select the dose of local anesthetic based upon the weight of the patient. This is a useful guideline, particularly in smaller patients and especially children; however, it would be unwise to follow this principle in obese patients. Just because a patient is obese does not necessarily mean that neural structures are any larger. The literature uses absolute numbers when publishing guidelines on safe doses of local anesthetics. There is no correlation between the weight of patients and the plasma concentration achieved after a given dose $\left(\mathrm{mg} \cdot \mathrm{kg}^{-1}\right)$ of local anesthetic. $^{7}$ The Compendium of Pharmaceuticals and Specialties recommends a maximum dose of $300 \mathrm{mg}$ of ropivacaine for major nerve blocks such as brachial plexus using a $0.75 \%$ concentration in adults. ${ }^{8}$ It is wise to adhere to these dosage recommendations when using potent local anesthetic agents such as bupivacaine and ropivacaine. We can be more flexible about dosage when using safer local anesthetics such as lidocaine, and the addition of epinephrine extends this flexibility even further. Excessive doses of ropivacaine were used in about one third of all the toxic reactions reported in the literature so far. The remainder involved accidental intravascular injections.

What is clear from these reports is that ropivacaineinduced cardiac toxicity is far more amenable to treatment than that reported with bupivacaine. Both patients who developed cardiac arrest responded rapidly to the usual methods of treatment and in one of these cases epinephrine was not even required. The patients with ventricular fibrillation also promptly responded to treatment and only one of them required defibrillation.

I have already alluded to a number of important preventive measures to reduce the likelihood of local anesthetic toxicity. There is some comfort in knowing that ropivacaine is safer than bupivacaine from a cardiac standpoint. However it is also important to know that bupivacaine is a more effective local anesthetic than ropivacaine when used in equal concentrations, meaning that a greater quantity of ropivacaine is required in order to achieve an equal effect. It is difficult to quantify the actual discrepancy because it seems to vary depending upon the concentration used. When used in lower concentrations this difference may be as much as $50 \%{ }^{9}$

How are cardiac toxic reactions to potent local anesthetics best treated? Weinberg recently published an excellent summary on this topic. ${ }^{10}$ There must be no delay in initiation of treatment of this condition. It is of paramount importance to prevent hypoxia, hypercarbia and metabolic acidosis and treat these conditions aggressively if they occur because they enhance toxic effects. ${ }^{11}$ This may require airway intervention and there should be no hesitation about administering a neuromuscular blocking drug to enable optimal airway management if necessary. Convulsions should be treated with small doses of benzodiazepines, thiopental or propofol. There should be no delay initiating cardiopulmonary resuscitation if indicated. There is strong support for the use of epinephrine and norepinephrine in low cardiac output states. However, these drugs may induce lethal ventricular arrhythmias. Vasopressin is a useful alternative in refractory cases. There is still some controversy about the use of phosphodiasterase inhibitors in local anesthetic induced cardiac depression. However, a recent study in animals compared milrinone and epinephrine in the treatment of ropivacaine induced cardiac depression and demonstrated that milrinone was superior. $^{12}$ Amiodarone is considered to be the treatment of choice for serious ventricular arrhythmias 
induced by potent local anesthetics. Calcium channel blockers are contraindicated as is phenytoin, and the use of bretylium is no longer supported. ${ }^{13}$ Cardiopulmonary bypass may be required in some cases, therefore it is important to alert the cardiac surgery team as early as possible. Potential new treatments for local anesthetic related cardiac toxicity include: lipid infusion, propofol and insulin/glucose/potassium infusions.

In summary, initial reports about bupivacaine induced cardiac toxicity were slow to emerge, as were those associated with ropivacaine. It is comforting to note that ropivacaine induced cardiac toxicity is far more readily treatable. Single shot epidural injections of high concentrations of bupivacaine in obstetric patients were responsible for a number of deaths in the past. Let us not make the same mistake with peripheral nerve blocks. Even though the majority of serious toxic reactions to local anesthetics occur following accidental intravascular injections, some occur because the recommended dose limits are exceeded. Therefore we should adhere to the dosage guidelines. Small incremental doses (no more than $5 \mathrm{~mL}$ at a time) of local anesthetic are recommended. Add a vascular marker to the local anesthetic and maintain verbal contact with the patient at all times. Finally, ECG monitoring is essential when performing regional anesthesia.

\section{La toxicité cardiaque à la ropivacaïne, com- parée à la bupivacaïne, ne présente pas autant de complications}

Les réactions toxiques graves aux anesthésiques locaux sont un problème depuis leur découverte par Koller en 1884. ${ }^{1}$ Des centaines d'anesthésiques locaux ont été testés au cours des 100 dernières années et très peu ont été utilisés en clinique. La lidocaïne, d'usage clinique depuis près de 60 ans, est toujours l'anesthésique local le plus utilisé dans le monde. C'est encore l'un des plus sûrs et efficaces jamais produits. L'une de ses principales limites est sa courte durée d'action. La bupivacaïne, adoptée en clinique en 1967, a représenté une nouvelle famille d'anesthésiques locaux - des dérivés de la pipécholoxylidine. Les recherches initiales sur l'innocuité et l'efficacité promettaient beaucoup. Anesthésique local à très longue action, il produit une anesthésie motrice et sensitive nettement séparées, contrairement aux anesthésiques locaux antérieurs. La bupivacaïne était utilisée depuis près de dix ans avant qu'on ne la relie à une grave toxicité cardiaque. Des décès chez des patientes d'obstétrique ont été rapportés aux États-Unis dans les années 1970.2 Des recherches pour remplacer la bupivacaïne ont commencé dans les années 1980. La ropivacaïne a été testée pour la première fois en 1988 et a montré de nombreuses caractéristiques anesthésiques de la bupivacaïne, mais était moins toxique. ${ }^{3}$ La majorité des anesthésiques locaux sont produits comme des mélanges racémiques. La ropivacaïne a été le premier anesthésique local produit synthétiquement qui était un énantiomère simple et, pour cette raison, moins toxique comme c'était prévisible. Son usage clinique a été approuvé en 1996 en Amérique du Nord. Les premiers articles sur une grave toxicité cardiaque avec la ropivacaïne ont été publiés en 2003. Deux patients ont subi un arrêt cardiaque et un patient a été victime de fibrillation ventriculaire après avoir reçu des doses excessives de ropivacaïne. ${ }^{4-6}$

Dans le présent numéro du Journal, un nouvel article rapporte de la fibrillation ventriculaire suivant l'injection intravasculaire accidentelle de ropivacaïne. ${ }^{7}$ Certains aspects intéressants de ce cas méritent d'être commentés. Ils illustrent clairement l'importance du monitorage avec l'usage d'anesthésiques locaux puissants. L'anesthésie régionale est souvent réalisée dans une «salle de bloc» ou une «aire d'attente» jouxtant les salles d'opération où l'accès à un monitorage complet n'est pas toujours disponible. Pour tous les patients devant recevoir une anesthésie régionale, il faudrait un monitorage de l'électrocardiogramme (ECG) et de la tension artérielle et la sphygmooxymétrie, comme dans le cas présent. Ce cas illustre aussi l'importance de la sédation et du fait d'avoir toute l'attention du patient au moment de réaliser l'anesthésie régionale.

Il est intéressant de noter que les quatre victimes de toxicité cardiaque grave, suivant l'injection de ropivacaïne, ont eu des blocs nerveux périphériques des membres inférieurs selon des techniques de stimulation nerveuse. Trois d'entre eux étaient âgés et ont reçu des solutions à $0,75 \%$ de ropivacaïne. Les réactions toxiques signalées étaient probablement causées par une injection intravasculaire accidentelle. Les blocs périphériques sont plus fréquemment utilisés à travers le monde pour une foule de raisons et la stimulation électrique sert souvent à préciser le siège anatomique 
de divers nerfs. On fait la plupart des injections avec des aiguilles pour bloc nerveux périphérique selon une technique d'injection unique. Une fois le nerf repéré grâce à la stimulation électrique, la position de l'aiguille est fixée et l'anesthésique local est injecté en prenant soin de ne pas bouger l'aiguille. On tend aussi à injecter plus rapidement l'anesthésique local dans ces circonstances de peur que le patient ne bouge comme le montre le cas de Gielen. ${ }^{7}$ C'est particulièrement important d'utiliser alors un marqueur cardiovasculaire (épinéphrine). La ropivacaïne est souvent injectée sans ajout d'épinéphrine, car elle n'est pas potentialisée par l'épinéphrine et la capture plasmatique de ropivacaïne est très peu touchée. ${ }^{8}$ L'épinéphrine n'a pas été ajoutée à la solution d'anesthésique local chez trois des victimes de toxicité cardiaque grave et elle a été utilisée en concentration réduite chez la quatrième (1:400,000). L'épinéphrine est un adjuvant essentiel de tous les anesthésiques locaux puissants pour l'anesthésie régionale majeure, sauf contre-indication.

La stimulation nerveuse n'a pas été universellement adoptée par les experts en anesthésie régionale. Des artisans expérimentés de la «vieille école» d'anesthésie régionale ne l'utilisent pas pour les blocs périphériques mais préfèrent se fier plutôt à la méthode de la paresthésie pour repérer le site du nerf. Ils inondent ensuite la région anatomique avec l'anesthésique local, déplaçant l'aiguille d'un mouvement de va-et-vient en éventail. Cette approche peut réduire la possibilité d'injection intravasculaire de doses importantes d'anesthésique local. Quelle que soit la technique, il faut administrer de petites doses progressives d'anesthésique local (pas plus de $5 \mathrm{~mL}$ à la fois), arrêter et établir un contact verbal avec le patient avant d'injecter une autre dose. Fait à noter, des techniques péridurales d'injection unique ont été fréquemment utilisées par les anesthésiologistes en obstétrique pour la césarienne il y a 25 ans, au moment où Albright a attiré l'attention sur la toxicité cardiaque liée à la bupivacaïne. $^{7}$

Il semble y avoir une certaine confusion parmi les anesthésiologistes sur ce qu'est une dose sûre de ropivacaïne. La plupart vont choisir la dose d'anesthésique local d'après le poids du patient. C'est utile, en particulier chez les petits patients et surtout les enfants ; mais il serait imprudent de suivre ce principe avec les patients obèses. Chez un patient obèse, les structures neurales ne sont pas nécessairement plus grosses. On retrouve des nombres absolus dans la publication de directives sur les doses sûres d'anesthésique local. Il n'y a pas de corrélation entre le poids du patient et la concentration plasmatique atteinte après une dose donnée $\left(\mathrm{mg} \cdot \mathrm{kg}^{-1}\right) \quad$ d'anesthésique local. $^{7} \quad$ Le
Compendium des produits et spécialités pharmaceutiques recommande une dose maximale de $300 \mathrm{mg}$ de ropivacaïne pour des blocs nerveux majeurs comme celui du plexus brachial où on utilise une concentration de $0,75 \%$ chez les adultes. ${ }^{8}$ Il est prudent d'administrer ce dosage pour un anesthésique local puissant comme la bupivacaïne et la ropivacaïne. Nous pouvons être plus souples avec des anesthésiques locaux plus sûrs comme la lidocaïne et l'addition d'épinéphrine ajoute encore plus de flexibilité. Des doses excessives de ropivacaïne ont été utilisées dans un tiers environ de toutes les réactions toxiques rapportées jusqu'à maintenant. Les autres réactions relèvent d'injections intravasculaires accidentelles.

Ce qui est certain à la lumière de ces articles, c'est que la toxicité cardiaque induite par la ropivacaïne est de loin plus facile à traiter que celle qui est provoquée par la bupivacaïne. Les deux patients qui ont subi un arrêt cardiaque ont réagi rapidement aux méthodes habituelles de traitement et dans l'un des cas, l'épinéphrine n'a même pas été nécessaire. Les patients qui présentaient de la fibrillation ventriculaire ont aussi répondu rapidement au traitement et un seul a eu besoin de défibrillation.

Nous avons déjà fait allusion à un certain nombre de mesures préventives importantes pour réduire la possibilité de toxicité à l'anesthésique local. Il peut être consolant de savoir que la ropivacaïne est plus sûre que la bupivacaïne d'un point de vue cardiaque. Il est aussi important de savoir que la bupivacaïne est une anesthésique local plus efficace que la ropivacaïne en concentration égale, ce qui veut dire qu'une plus grande quantité de ropivacaïne est nécessaire pour obtenir un même effet. Il est difficile de quantifier l'écart réel parce qu'il semble varier en fonction de la concentration utilisée. Pour des concentrations faibles, cet écart peut aller jusqu'à $50 \%{ }^{9}$

Comment les réactions cardiaques toxiques aux anesthésiques locaux puissants sont-elles le mieux traitées ? Weinberg a récemment publié un excellent résumé sur le sujet. ${ }^{10}$ On doit traiter ce problème sans tarder. Il faut prévenir l'hypoxie, l'hypercapnie et l'acidose métabolique et traiter ces désordres agressivement, s'ils surviennent, parce qu'ils accentuent les effets toxiques. ${ }^{11}$ La situation peut exiger une intervention sur les voies aériennes et on ne devrait pas hésiter à administrer un myorelaxant pour faciliter le contrôle optimal des voies aériennes au besoin. Les convulsions doivent être traitées avec de petites doses de benzodiazépines, de thiopental ou de propofol. On devrait amorcer sans délai la réanimation cardiopulmonaire, si indiqué. On encourage fortement l'usage d'épinéphrine et de norépinéphrine en cas de faible 
débit cardiaque. Cependant, ces médicaments peuvent induire des arythmies ventriculaires mortelles. La vasopressine peut être un autre bon choix dans les cas réfractaires. On discute toujours au sujet de l'utilisation d'inhibiteurs de phosphodiastérase en cas de dépression cardiaque provoquée par un anesthésique local. Mais une étude récente chez des animaux a comparé la milrinone et l'épinéphrine comme traitement de la dépression cardiaque induite par la ropivacaïne et démontré que la milrinone était supérieure. ${ }^{12}$ L'amiodarone est considérée comme le meilleur traitement pour les arythmies ventriculaires graves induites par des anesthésiques locaux puissants. Les inhibiteurs calciques sont contre-indiqués, de même que la phénytoïne, et l'usage de brétylium n'est plus soutenu. $^{13}$ La circulation extracorporelle peut être nécessaire dans certains cas, il faut alors prévenir l'équipe de chirurgie cardiaque au plus tôt. Les nouveaux traitements possibles pour la toxicité cardiaque reliée à l'anesthésique local comprennent : une perfusion lipidique, du propofol et des perfusions d'un mélange d'insuline, de glucose et de potassium.

En résumé, les premiers articles sur la toxicité cardiaque induite par la bupivacaïne ont été lents à paraître, comme l'ont été ceux sur la toxicité liée à la ropivacaïne. Il est réconfortant de savoir que la toxicité cardiaque induite par la ropivacaïne est de loin plus facile à traiter. Des injections péridurales uniques de fortes concentrations de bupivacaïne chez les patientes d'obstétrique ont été responsables d'un certain nombre de décès dans le passé. Ne répétons pas l'erreur avec les blocs périphériques. Même si la majorité des réactions toxiques graves aux anesthésiques locaux surviennent après des injections intravasculaires accidentelles, certaines relèvent de doses excessives. Il faut donc suivre les recommandations de dosage, c'est-àdire des petites doses incrémentielles (pas plus de 5 $\mathrm{mL}$ à la fois). Ajouter un marqueur vasculaire à l'anesthésique local et maintenir le contact verbal avec le patient en tout temps. Finalement, le monitorage de l'ECG est essentiel avec l'anesthésie régionale.

\section{References}

1 Koller $C$. Historical notes on the beginning of local anesthesia. JAMA 1928; 90: 1742-3.

2 Hawkins JL, Koonin LM, Palmer SK, Gibbs CP. Anesthesia-related deaths during obstetric delivery in the United States, 1979-1990. Anesthesiology 1997; 86: 277-84.

3 Akerman B, Hellberg IB, Trossvik C. Primary evaluation of the local anaesthetic properties of the amino amide agent ropivacaine (LEA 103). Acta Anaesthesiol Scand 1988; 32: 571-8.
4 Huet O, Eyrolle LJ, Mazoit JX, Ozier YM. Cardiac arrest after injection of ropivacaine for posterior lumbar plexus blockade. Anesthesiology 2003; 99: 1451-3.

5 Chazalon P, Tourtier JP, Villevielle T, et al. Ropivacaine-induced cardiac arrest after peripheral nerve block: successful resuscitation. Anesthesiology 2003; 99: 1449-51.

6 Klein SM, Pierce T, Rubin $\Upsilon$, Nielsen KC, Steele SM. Successful resuscitation after ropivacaine-induced ventricular fibrillation. Anesth Analg 2003; 97: 901-3.

7 Gielen M, Slappendel R, Jack N. Successful defibrillation immediately after the intravascular injection of ropivacaine. Can J Anesth 2005; 52: 490-2.

8 Hickey R, Blanchard J, Hoffman J, Sjovall J, Ramamurthy $S$. Plasma concentrations of ropivacaine given with or without epinephrine for brachial plexus block. Can J Anaesth 1990; 37: 878-82.

9 Polley LS, Columb MO, Naughton NN, Wagner DS, van de Ven CJ. Relative analgesic potencies of ropivacaine and bupivacaine for epidural analgesia in labor. Implications for therapeutic indexes. Anesthesiology 1999; 90: 944-50.

10 Weinberg GL. Current concepts in resuscitation of patients with local anesthetic cardiac toxicity. Reg Anesth Pain Med 2002; 27: 568-75.

11 Feldman HS, Arthur GR, Pitkanen M, Hurley R, Doucette AM, Covino BG. Treatment of acute systemic toxicity after rapid intravenous injection of ropivacaine and bupivacaine in the conscious dog. Anesth Analg 1991; 73: 373-84.

12 Neustein S, Sampson I, Dimich I, Shiang H, Tatu J. Milrinone is superior to epinephrine as treatment of myocardial depression due to ropivacaine in pigs. Can J Anesth 2000; 47: 1114-8.

13 Tallman RD Jr, Rosenblatt RM, Weaver JM, Wang $\Upsilon$. Verapamil increases the toxicity of local anesthetics. J Clin Pharmacol 1988; 28: 317-21. 